\title{
Robotic assisted right middle lobectomy
}

\author{
Waël C. Hanna \\ Division of Thoracic Surgery, McMaster University, Hamilton, ON, Canada \\ Correspondence to: Waël C. Hanna, MDCM, MBA, FRCSC. St. Joseph’s Healthcare Hamilton, 50 Charlton Ave. E, Juravinski Tower, Suite T2105 F, \\ Hamilton, ON L8N4A6, Canada. Email: hannaw@mcmaster.ca. \\ Provenance: This is an invited Editorial commissioned by Section Editor of fTD, Jianfei Shen, MD (Department of Cardiothoracic Surgery, Taizhou \\ Hospital of Zhejiang Province, Wenzhou Medical University, Taizhou, China). \\ Comment on: Chen X, Yang S, Guo W, et al. Robotic-assisted right middle lobectomy. AME Med J 2017;2:8.
}

Submitted Jul 25, 2017. Accepted for publication Jul 31, 2017.

doi: $10.21037 /$ jtd.2017.08.38

View this article at: http://dx.doi.org/10.21037/jtd.2017.08.38

Dr. Chen and colleagues share their experience in performing a right middle lobectomy (RML) for a small peripheral ground-glass tumor (1). They use a 5-port approach: 3 robotic arms, 1 assistant and one camera. The incisions are placed between the $5^{\text {th }}$ and $8^{\text {th }}$ intercostal space, and the procedure is done through an anterior hilar approach, with vein divided first, followed by pulmonary artery, followed by bronchus. Lastly, the posterior mediastinal pleura was dissected and lymph node station 7 was harvested.

RML lobectomies are interesting operations. When the lesion is small and peripheral, the operation is usually straightforward and low-risk. However, when the tumor is bulky, or central on the fissure, these operations can be complicated, requiring meticulous vascular dissection around the pulmonary artery. At our institution, we use a different approach, which may facilitate RML lobectomy when it is a complicated operation. We use a 5 -port technique according to the CPRL-4 positioning described by Cerfolio (2). We find that the ports all lining up in the same interspace minimizes postoperative pain and allows for better visualization of the fissure then the camera is looking down on pulmonary artery. We also start every robotic case with a posterior mediastinal incision and dissection of lymph node stations 9, 7, 4R, 2R and 11RS. This delivers the lung from the rigidity of the posterior mediastinum and renders the hilar dissection around the vessels much easier. We then divide the RML vein, followed by pulmonary artery, followed by bronchus and finally the fissure.

Regardless of which approach is taken, it is important for robotic teams to be consistent in the way they perform robotic setup, docking, and operating. Repetition allows for streamlining of manoeuvres, improves operating room efficiency and minimizes complications. With the advent of low dose computed tomography, we expect a surge in minimally invasive targeted surgery for small lung tumors, and robotic surgeons will be on the forefront of this emerging field.

\section{Acknowledgements}

None.

\section{Footnote}

Conflicts of Interest: The author has no conflicts of interest to declare.

\section{References}

1. Chen X, Yang S, Guo W, et al. Robotic-assisted right middle lobectomy. AME Med J 2017;2:8.

2. Cerfolio RJ, Bryant AS. How to teach robotic pulmonary resection. Semin Thorac Cardiovasc Surg 2013;25:76-82.
Cite this article as: Hanna WC. Robotic assisted right middle lobectomy. J Thorac Dis 2017;9(8):2325. doi: 10.21037/ jtd.2017.08.38 